Research, Society and Development, v. 9, n. 4, e189943016, 2020

(CC BY 4.0) | ISSN 2525-3409 | DOI: http://dx.doi.org/10.33448/rsd-v9i4.3016

Consumo de drogas lícitas e ilícitas por estudantes universitários

Consumption of licit and illicit drugs by university students

Consumo de ley y drogas ilegales por estudiantes universitários

Recebido: 05/03/2020 | Revisado: 07/03/2020 | Aceito: 17/03/2020 | Publicado: 20/03/2020

\title{
Christian Barbosa de Freitas
}

ORCID: https://orcid.org/ 0000-0002-3206-619X

Centro Universitário Metropolitano da Amazônia, Brasil

E-mail: christianfarmaceutico@gmail.com

Thiago Casanova Pereira Veloso

ORCID: https://orcid.org/ 0000-0001-7642-1117

Centro Universitário Metropolitano da Amazônia, Brasil

E-mail: casanova@veloxmail.com.br

Lenoilson Passos da Silva Segundo

ORCID: https://orcid.org/ 0000-0002-7829-2184

Centro Universitário Metropolitano da Amazônia, Brasil

E-mail: lenonsegundo@gmail.com

Filipe Pereira Gonçalo de Sousa

ORCID: https://orcid.org/0000-0003-4901-3876

Centro Universitário Metropolitano da Amazônia, Brasil

E-mail: filipe_gonçalo1992@hotmail.com

Brenda Sussuarana Galvão

ORCID: https://orcid.org/ 0000-0003-3435-2373

Centro Universitário Metropolitano da Amazônia, Brasil

E-mail: brenda_sussu@hotmail.com

Phelipe Augusto Rabelo Paixão

ORCID: https://orcid.org/ 0000-0002-7642-1659

Centro Universitário Metropolitano da Amazônia, Brasil

E-mail: phelipepaixao@hotmail.com

\section{Resumo}

Este estudo tem como objetivo analisar o consumo de drogas lícitas e ilícitas por estudantes universitários. Trata-se de uma revisão integrativa, utilizou-se de 12 artigos disponíveis nas 
seguintes bases de dados: Scielo, Pubmed e Lilacs. Os critérios de inclusão foram: pesquisas que abordassem a temática, publicadas em inglês, português ou espanhol em formato de artigos, teses e dissertações no período de 2012 a 2019. Como critérios de exclusão: trabalhos que não apresentassem resumos na íntegra nas bases de dados e não apresentasse caráter cientifico. Em todos os estudos analisados verificou-se que os estudantes universitários em sua maioria fazem uso de drogas, e que a maioria relatou consumo de álcool e tabaco, mais também se observou o consumo de diversas drogas ilícitas como a maconha, cocaína, entre outras. Sugere-se que haja um incentivo ao desenvolvimento de políticas públicas voltadas a este público, que diminuíam a vulnerabilidade destes estudantes. Tendo em vista que o consumo destas substâncias pode trazer prejuízos a sua saúde e acarretar outros problemas durante a fase adulta. Palavras-chave: Drogas ilícitas. Universitários. Saúde Pública.

\begin{abstract}
This study aims to analyze the consumption of licit and illicit drugs by university students. It is an integrative review, using 12 articles available in the following databases: Scielo, Pubmed and Lilacs. The inclusion criteria were: research that addressed the theme, published in English, Portuguese or Spanish in the form of articles, theses and dissertations in the period from 2012 to 2019. As exclusion criteria: papers that did not present full abstracts in the databases and was not of a scientific nature. In all the analyzed studies, it was verified that the university students mostly use drugs, and that most reported alcohol and tobacco consumption, but also observed the consumption of several illicit drugs such as marijuana, cocaine, among others. It is suggested that there is an incentive to the development of public policies aimed at this public, which diminished the vulnerability of these students. Considering that the consumption of these substances can damage your health and cause other problems during adulthood.
\end{abstract}

Keywords: Illicit drugs. College students. Public health.

\title{
Resumen
}

Este estudio tiene como objetivo analizar el consumo de drogas lícitas e ilícitas por estudiantes universitarios. Se trata de una revisión integrativa, se utilizó de 12 artículos disponibles en las siguientes bases de datos: Scielo, Pubmed y Lilacs. Los criterios de inclusión fueron: estudios que abordaron el tema, publicado en formato portugués o español de artículos, tesis y disertaciones en los 2.012 periodo de 2019. Los criterios de exclusión Inglés,: trabajos que no presenten los resúmenes en su totalidad en las bases de datos y no presentase carácter científico. En todos los estudios analizados se verificó que los estudiantes universitarios en su mayoría 
hacen uso de drogas, y que la mayoría relató consumo de alcohol y tabaco, más también se observó el consumo de diversas drogas ilícitas como la marihuana, cocaína, entre otros. Se sugiere que haya un incentivo al desarrollo de políticas públicas dirigidas a este público, que disminuían la vulnerabilidad de estos estudiantes. Teniendo en cuenta que el consumo de estas sustancias puede traer perjuicios a su salud y acarrear otros problemas durante la fase adulta.

Palabras clave: Drogas ilícitas. Universidad. Salud pública.

\section{Introdução}

No Brasil, o uso e/ou abuso de drogas sejam elas lícitas como o álcool, tabaco e medicações ou ilícitas como crack, maconha, anfetaminas entre outras, apresentam uma relação direta e indireta com uma série de agravos à saúde, como agressões, acidentes de trânsito, distúrbios de conduta, comportamento de risco no âmbito sexual, transmissão do vírus HIV pela a utilização de drogas injetáveis, além de outros problemas de saúde decorrentes dos componentes advindos das substâncias utilizadas e das vias de administração, como o álcool associado à cirrose, e o cigarro pelo fato de conter nicotina, associado ao câncer de pulmão, entre outros (Zeferino \& Fermo, 2012).

Atualmente, há um excesso no uso de drogas por diversos grupos e classes. Um dos segmentos sociais que chama a atenção da comunidade científica é o dos universitários. Eles diariamente encaram situações que compõem um processo especial já que muitos deles estão em um ciclo de vida que traz consigo uma série de conflitos tanto cognitivos como afetivos e, se não tratados adequadamente, podem sair do controle (Zeferino \& Hamilton, 2015).

Para Nóbrega (2012), o abuso de substâncias deve ser compreendido por uma visão multifatorial. Logo então, existem diversos fatores predisponentes que estão ligados estatisticamente ao aumento da probabilidade do abuso de drogas. Entre estes, os fatores socioculturais, no qual o jovem vive em um ambiente onde amigos e familiares consomem substâncias psicoativas e ele por curiosidade, imitação ou por pressão de outros passa a consumir também, muitas vezes para se sentir pertencente a determinado grupo.

Nas últimas décadas, o consumo de álcool vem aumentando em todo o mundo, considerado um dos maiores problemas de saúde pública atual. Quando se refere ao consumo do álcool relacionado com estudantes, alguns dados são mais preocupantes. Isso devido ao álcool ser uma das poucas drogas psicotrópicas que têm seu uso legalizado e incentivado pela sociedade. Estudo realizado com estudantes mostra que, para eles, o alcoolismo estaria ligado à fuga de problemas cotidianos, para lidar e fugir de situações negativas de caráter pessoal, em 
decorrência de pressão social direta ou indireta, alternativa para lidar com situações negativas resultantes de aspectos sociais e econômicos e, principalmente, pelo caráter prazeroso que a bebida oferece ou da situação em que é consumida (Barros et al., 2012).

Diante do que foi exposto o presente estudo tem como objetivo conhecer mais sobre o consumo de drogas lícitas e ilícitas por estudantes universitários, de acordo com os dados disponíveis na literatura.

\section{Metodologia}

Este artigo trata-se de uma revisão de literatura do tipo integrativa. Para busca e seleção dos estudos foram utilizadas as seguintes bases de dados: Scielo, Pubmed e Lilacs, totalizando 12 artigos. A tabela 01 permite uma visualização dos descritores que foram utilizados para a construção da referida revisão de literatura.

Tabela 01: Descritores utilizados para a construção da revisão de literatura.

\begin{tabular}{|c|c|c|c|c|c|c|}
\hline \multirow{2}{*}{ DESCRITORES } & \multicolumn{2}{c}{ SCIELO } & \multicolumn{2}{c}{ PUBMED } & \multicolumn{2}{c}{ LILACS } \\
\cline { 2 - 8 } & Total & Seleção & Total & Seleção & Total & Seleção \\
\hline Drogas ilícitas & 196 & 03 & 26.599 & 03 & 1704 & 02 \\
\hline Universitários & 1.449 & 01 & 133.259 & 0 & 6.426 & 01 \\
\hline Saúde publica & 14.056 & 01 & 144.567 & 0 & 12.456 & 01 \\
\hline
\end{tabular}

Fonte: Dados da pesquisa.

Os critérios de inclusão foram: pesquisas que abordassem a temática, publicadas em inglês, português ou espanhol em formato de artigos, teses e dissertações no período de 2012 a 2019. Como critérios de exclusão: trabalhos que não apresentassem resumos na íntegra nas bases de dados e não apresentasse caráter cientifico.

\section{Resultados e discussão}

A tabela 02 apresenta a síntese dos estudos selecionados para a construção da revisão de literatura.

Tabela 02: Síntese dos estudos selecionados para a construção da revisão de literatura. 


\begin{tabular}{|c|c|c|c|}
\hline Artigo & Ano & Autores & Amostra \\
\hline $\begin{array}{c}\text { O uso de álcool e tabaco entre } \\
\text { acadêmicos de uma universidade do Sul } \\
\text { do Brasil. }\end{array}$ & 2017 & Ferraz et al. & $\begin{array}{l}284 \text { acadêmicos de } \\
\text { Medicina, Direito e } \\
\text { Engenharia Civil. }\end{array}$ \\
\hline $\begin{array}{c}\text { Consumo de álcool e outras drogas em } \\
\text { uma Faculdade de Medicina do sul de } \\
\text { Minas Gerais. }\end{array}$ & 2016 & $\begin{array}{l}\text { Tostes; } \\
\text { Campos \& } \\
\text { Pereira. }\end{array}$ & $\begin{array}{l}419 \text { acadêmicos de } \\
\text { Medicina. }\end{array}$ \\
\hline $\begin{array}{l}\text { Condutas de saúde de universitários } \\
\text { ingressantes e concluintes de cursos da } \\
\text { área da saúde }\end{array}$ & 2016 & $\begin{array}{l}\text { Campos et } \\
\text { al. }\end{array}$ & $\begin{array}{l}323 \text { acadêmicos das } \\
\text { diversas áreas de } \\
\text { conhecimento. }\end{array}$ \\
\hline $\begin{array}{l}\text { Consumo de drogas entre estudantes } \\
\text { universitários: família, espiritualidade e } \\
\text { entretenimento moderando a influência } \\
\text { dos pares. }\end{array}$ & 2015 & $\begin{array}{l}\text { Zeferino et } \\
\text { al. }\end{array}$ & $\begin{array}{l}250 \text { acadêmicos da área de } \\
\text { Ciências da Saúde e } \\
\text { Ciências Sociais/Humanas. }\end{array}$ \\
\hline $\begin{array}{c}\text { Uso de álcool e outras drogas por } \\
\text { estudantes universitários de uma região } \\
\text { de fronteira do Sul do Brasil. }\end{array}$ & 2015 & $\begin{array}{l}\text { Priotto; } \\
\text { Lizzi \& } \\
\text { Nihei. }\end{array}$ & $\begin{array}{l}760 \text { acadêmicos das } \\
\text { diversas áreas de } \\
\text { conhecimento. }\end{array}$ \\
\hline $\begin{array}{l}\text { Perfil dos estudantes de uma instituição } \\
\text { de ensino superior quanto ao uso de } \\
\text { álcool e outras drogas. }\end{array}$ & 2015 & Freitas et al & $\begin{array}{c}84 \text { dos cursos de } \\
\text { Enfermagem, Fisioterapia } \\
\text { e Nutrição. }\end{array}$ \\
\hline $\begin{array}{l}\text { Implicações do uso de álcool, tabaco e } \\
\text { outras drogas na vida do universitário. }\end{array}$ & 2015 & $\begin{array}{l}\text { Junior \& } \\
\text { Gaya. }\end{array}$ & $\begin{array}{l}123 \text { acadêmicos das } \\
\text { diversas áreas de } \\
\text { conhecimento. }\end{array}$ \\
\hline $\begin{array}{l}\text { Perfil do consumo de álcool, tabaco e } \\
\text { maconha entre graduandos em } \\
\text { Enfermagem da Faculdade de Educação } \\
\text { e MeioAmbiente - Faema. }\end{array}$ & 2014 & $\begin{array}{l}\text { Vale; } \\
\text { Uesugui \& } \\
\text { Pereira. }\end{array}$ & $\begin{array}{l}137 \text { acadêmicos de } \\
\text { Enfermagem. }\end{array}$ \\
\hline $\begin{array}{l}\text { Levantamento domiciliar sobre o uso de } \\
\text { substâncias psicotrópicas ilícitas entre } \\
\text { estudantes de uma universidade do alto } \\
\text { tietê. }\end{array}$ & 2014 & $\begin{array}{c}\text { Dias \& } \\
\text { Conceição. }\end{array}$ & $\begin{array}{l}612 \text { acadêmicos das } \\
\text { diversas áreas do } \\
\text { conhecimento. }\end{array}$ \\
\hline $\begin{array}{l}\text { Uso de álcool e tabaco entre estudantes } \\
\text { de Psicologia da Universidade Federal } \\
\text { do Espírito Santo. }\end{array}$ & 2013 & $\begin{array}{l}\text { Santos; } \\
\text { Pereira \& } \\
\text { Siqueira. }\end{array}$ & $\begin{array}{l}221 \text { acadêmicos de } \\
\text { Psicologia. }\end{array}$ \\
\hline
\end{tabular}


(CC BY 4.0) | ISSN 2525-3409 | DOI: http://dx.doi.org/10.33448/rsd-v9i4.3016

Policonsumo simultâneo de drogas entre estudantes de graduação da área de ciências da saúde de uma universidade: implicações de gênero, sociais e legais, Santo André - Brasil.
275 acadêmicos dos cursos de Enfermagem, Medicina,

Farmácia, Nutrição, Fisioterapia e Terapia Ocupacional.

560 acadêmicos das diversas áreas do conhecimento.

Fonte: Dados da pesquisa.

Em estudo realizado por Ferraz et al (2017), com a participação de 284 acadêmicos de Medicina, Direito e Engenharia Civil através da aplicação de um questionário, verificou que dos estudantes participantes 85,2\% ( $\mathrm{n}=242)$ consumiam álcool e 18,7\% ( $\mathrm{n}=53)$ tabaco. Em relação ao consumo das substâncias e o sexo dos universitários, não houve diferença. Porém, em relação ao tabaco, evidenciou-se que os homens consomem mais que as mulheres.

Tostes; Campos \& Pereira (2016), em seus estudos com 419 acadêmicos do curso de medicina através de um questionário anônimo, obtiveram como resultados que no mês anterior a pesquisa, 87,6\% (n=367) usaram álcool, 3,4\% $(\mathrm{n}=98)$ fizeram uso de tabaco, 13,4\% (n=56) usaram maconha, estimulantes, 6,9\% $(\mathrm{n}=29)$, tranquilizantes, 6,2\% $(\mathrm{n}=26)$, inalantes, 5,5\% $(n=23)$ alucinógenos, 4,8\% ( $n=20)$ cocaína /crack, 1,7\% (n=7), opiáceos 0,2\% (n=1).

Campos et al (2016), em seu estudo comparando 323 acadêmicos do primeiro e último período verificou que em relação ao consumo de tabaco os alunos iniciantes referiram 14,86\% $(n=48)$ e os veteranos $26,14 \%$ ( $n=46)$, em relação ao uso de álcool os calouros evidenciaram $56,96 \%(\mathrm{n}=184)$ e os veteranos $75 \%(\mathrm{n}=132)$, ao avaliarem o uso de maconha verificou -se que os calouros e veteranos apresentaram $11,76 \%(n=38)$ e $21,59 \%(n=38)$, respectivamente.

Zeferino et al (2015), em estudo com 250 acadêmicos, verificando o consumo de drogas licitas e ilícitas durante os 12 últimos meses, constataram que a droga lícita mais consumida foi o álcool correspondendo a 91,6\% ( $n=229)$ e em segundo lugar o tabaco 34,4\% ( $n=86)$. Em relação as drogas ilícitas a mais consumida foi a maconha representando 30,4\% (n=76) e logo após o ecstasy 2,8\% (n=7) da amostra.

Em estudo realizado por Priotto; Lizzi \& Nihei (2015), com 760 acadêmicos das diversas áreas, verificou-se que 26,1\% experimentaram algum tipo de droga e 89,5\% usaram álcool. O tabaco $23,6 \%$ e maconha $11,9 \%$ foram as drogas mais relatadas pelos mesmos.

No estudo de Freitas et al (2015), em relação às drogas mais consumidas entre os 84 universitários e sua frequência de uso observou-se que a droga mais consumida nos últimos 30 
dias referente à data de preenchimento do questionário, foi o álcool com 56\% ( $\mathrm{n}=47)$. Seguida pelo cigarro, onde $10,7 \%(n=9)$ afirmaram ter fumado de um a dez cigarros por dia. A maconha foi a terceira 8,3\% $(\mathrm{n}=7)$ fumaram de uma a cinco vezes por dia. Logo após as substâncias inalatórias (loló, lança perfume,etc.) 7,1\% (n=6) disseram ter usado de uma a cinco vezes por dia. Em quinto lugar ficou a cocaína com 2,4\% $(n=2)$ que usaram de uma a cinco vezes por dia. Em sexto lugar o LSD (dietilamida do ácido lisérgico) e o Chá de Cogumelo com 3,6\% (n= 3) afirmaram ter usado de uma a cinco vezes por dia. Por fim o êxtase (ecstasy) em que 1,2\% $(n=1)$ afirmaram ter feito o uso de uma a cinco vezes por dia.

Junior \& Gaya (2015) em pesquisa realizada com 123 acadêmicos das diversas áreas através de um questionário, obteve como resultados que 89,4\% (n=110) dos universitários eram consumidores de bebidas alcoólicas, 42,3\% $(n=52)$ usavam tabaco e 20,3\% $(n=25)$ faziam o uso de maconha. O uso de inalantes, hipnóticos e/ou sedativos foi relatado por 10,6\% $(\mathrm{n}=13)$ dos participantes, e o uso de cocaína e crack por 8,9\% (n=11) dos estudantes.

No estudo de Vale; Uesugui \& Pereira (2014), onde utilizou-se como amostra 137 acadêmicos de Enfermagem devidamente matriculados na referida instituição. Do total de 137 acadêmicos, 113 indivíduos relataram que consumiram drogas, sendo que 58\% (n=66) relataram consumo de apenas álcool, 27\% $(\mathrm{n}=30)$ consumo de álcool e tabaco, 14\% (n=16) álcool, tabaco e maconha e $1 \%(n=1)$ referiram consumo de álcool e maconha.

Dias \& Conceição (2014), em estudo realizado com 612 acadêmicos de diversas áreas em relação ao uso de drogas ilícitas, observou que um mês anterior a pesquisa $5 \%$ relataram o uso de maconha. Logo após os tranquilizantes com 3\%, seguidos pela cocaína com 1,29\%, alucinógenos com $0,66 \%$, esteroides e inalantes $0,37 \%$, anfetaminas e opiáceos $0,29 \%$.

Em pesquisa realizada por Santos; Pereira \& Siqueira (2013), com 221 acadêmicos de Psicologia através de aplicação de um questionário. Onde obteve-se como resultados no próprio mês desse estudo, 52,49\% ( $\mathrm{n}=16)$ relataram uso de álcool, 13,12\% $(\mathrm{n}=29)$ fizeram utilização de tabaco, 9,05\% $(\mathrm{n}=20)$ relataram o uso de maconha, 3,62\% $(\mathrm{n}=8)$ fizeram uso de tranquilizantes, $1,36 \%(\mathrm{n}=3)$ utilizaram alucinógenos, anfetamínicos e analgésicos opiáceos, 0,9\% (n=2) fizeram uso de codeína e cocaína e $0,45 \%(n=1)$ esteroides anabolizantes.

No estudo de Nóbrega et al (2012) procuravam avaliar o policonsumo simultâneo de drogas (PCSD) com 275 acadêmicos, verificou-se que a combinação álcool + cannabis foi feita por $30 \%$ nos últimos 12 meses, seguido da combinação de álcool + medicamentos prescritos realizados por $22 \%$, a combinação de álcool + tabaco + cannabis feita por $16 \%$, a combinação de tabaco + cannabis feita por $8 \%$. Outras combinações feitas nos últimos 12 meses álcool + tabaco + inalantes $4 \%$, e álcool + tabaco + drogas prescritas feito por $7 \%$. 
Medeiros et al (2012), em seu estudo realizado com 560 acadêmicos das diversas áreas, observaram que nos 3 meses anteriores a pesquisa, a droga mais consumida foi o álcool 71,4\%, logo após o tabaco com 22,8\%, em terceiro lugar a maconha $9,0 \%$, na quarta posição ficou as anfetaminas e ecstasy com $4,6 \%$, ainda foi relatado o consumo de hipnóticos ou sedativos $3,1 \%$, cocaína ou crack $2,8 \%$, inalantes e alucinógenos $1,8 \%$ e opioides $0,3 \%$.

Segundo Filho \& Teixeira (2012), o ingresso na universidade é o momento de maior vulnerabilidade para os estudantes, devido, principalmente, à vivência de novas experiências, a ausência da família e as novas amizades. Ressalta-se que as características socioambientais podem influenciar o uso excessivo de drogas e a ocorrência de comportamentos de risco. $\mathrm{O}$ meio acadêmico pode favorecer o uso, em decorrência de inúmeras festas contendo álcool e outras drogas e da pressão social para o consumo dessas substâncias.

Para os universitários, os efeitos do uso de bebidas alcoólicas e outros tipos de drogas são bastante sedutores e satisfatórios, uma vez que o álcool é percebido como um facilitador social, por aumentar a sensação de auto adequação e diminuir a ansiedade. Estudos apontam que as expectativas relacionadas ao uso de álcool e outras drogas estão vinculadas ao aumento da autoconfiança, da sociabilidade, descontração e desinibição social (PORTO et al., 2012).

Verifica-se que, cada vez mais, o consumo de drogas tem se tornado presente no meio universitário. Pesquisas revelam que estudantes universitários apresentam índices mais elevados de consumo do que a população de forma geral. Segundo dados da Secretaria Nacional de Políticas sobre Drogas, $80 \%$ dos estudantes universitários afirmaram ter consumido algum tipo de bebida alcoólica e 49\% experimentaram alguma droga ilícita pelo menos uma vez na vida (Marangoni \& Oliveira, 2012).

\section{Conclusão}

De acordo com os estudos conclui-se que o consumo de drogas lícitas ou ilícitas é mais frequente entre universitários do que na população em geral. Recomenda-se que haja o desenvolvimento de novos estudos nesta linha de investigação, a fim de proporcionar subsídios às políticas de promoção da saúde junto aos estudantes, evitando com isso, o agravamento dos casos, que muitas vezes pode levar ao baixo rendimento acadêmico, além de outras graves consequências sociais, bem como prejuízos a saúde e outros problemas durante a fase adulta.

\section{Referências}


Barros, C. L. et al. (2012). A influência do convívio universitário na adesão ao alcoolismo. Revista Eletrônica do Curso de Pedagogia do Campus Jataí - UFG, 2(13).

Campos, L. et al. (2016). Condutas de saúde de universitários ingressantes e concluintes de cursos da área da saúde. Rev. Bras. Pesq. Saúde, 18(2), 17-25.

Dias, A. A \& Conceição, A. S. (2014). Levantamento domiciliar sobre o uso de substâncias psicotrópicas ilícitas entre estudantes de uma universidade do alto tietê. Revista Saúde e Pesquisa, 7(3), 465-476.

Ferraz, L. et al. (2017). O uso de álcool e tabaco entre acadêmicos de uma universidade do sul do brasil. Rev Bras Promoç Saúde, 30(1), 79-85.

Filho, N. H \& Teixeira, M. A. P. (2012). Motivos para beber e situações de consumo de bebidas alcoólicas: um estudo explortatório. Mudanças, 20(2), 1-6.

Freitas, M. A. et al. Perfil dos estudantes de uma instituição de ensino superior quanto ao uso de álcool e outras drogas. Revista Ciência Plural, 1(2), 29-36.

Júnior, G. A \& Gaya, C. M. (2015). Implicações do uso de álcool, tabaco e outras drogas na vida do universitário. Rev Bras Promoç Saúde, 28(1), 67-74.

Marangoni, S. R \& Oliveira, M. L. F. O. (2012). Uso de Crack por multípara em vulnerabilidade social: história de vida. Ciênc Cuid Saúde, 11(1), 166-172.

Medeiros, S. B. et al. (2012). Prevalência do uso de drogas entre acadêmicos de uma universidade particular do sul do brasil. Aletheia, 1(39).

Nóbrega, M. P. S. S. (2012). Fenômeno das drogas lícitas e ilícitas e assistência de enfermagem. São Paulo: Rideel.

Nóbrega, M. P. S. et al. (2012). Policonsumo simultâneo de drogas entre estudantes de graduação da área de ciências da saúde de uma universidade: implicações de gênero, sociais e legais, Santo André - Brasil. Texto Contexto Enferm, 21(1).

Porto, G. M. et al. (2012). Uso ocasional, abusivo o dependência de sustancias psicoactivas entre los estudiantes de un curso de graduación em fisioterapia. Rev Dig EFDeportes.com, 17(170).

Priotto, E. M. T. P.; Lizzi, E. A. S \& Nihei, O. K. (2015). Uso de álcool e outras drogas por estudantes universitários de uma região de fronteira do sul do brasil. Revista espaço para a saúde, 16(4), 07-19.

Santos, M. V. F.; Pereira, D. S \& Siqueira, M. M. (2013). Uso de álcool e tabaco entre estudantes de Psicologia da Universidade Federal do Espírito Santo. J Bras Psiquiatr, 62(1), 22-30.

Tostes, J. G.; Campos, F. P \& Pereira, L. G. R. (2016). Consumo de álcool e outras drogas em uma Faculdade de Medicina do sul de Minas Gerais. Revista Ciências em Saúde, 6(2). 
Vale, J. D.; Uesugui, J. H. M \& Pereira, R. (2014). Perfil do consumo de álcool, tabaco e maconha entre graduandos em enfermagem da faculdade de educação e meio ambiente Faema. Revista Científica FAEMA, 5(2), 156-172.

Zeferino, M. T. et al. (2015). Consumo de drogas entre estudantes universitários: família, espiritualidade e entretenimento moderando a influência dos pares. Texto Contexto Enferm, $15(1), 125-135$.

Zeferino, M. T \& Fermo, V. C. (2012). Prevenção ao uso/abuso de drogas. Saúde do Adulto, 2(1), 9-42. p. 9-42.

\title{
Porcentagem de contribuição de cada autor no manuscrito
}

\author{
Christian Barbosa de Freitas - 15\% \\ Thiago Casanova Pereira Veloso - 15\% \\ Lenoilson Passos da Silva Segundo - 15\% \\ Filipe Pereira Gonçalo de Sousa - 15\% \\ Brenda Sussuarana Galvão-15\% \\ Phelipe Augusto Rabelo Paixão - 25\%
}

